\title{
An illusion of ingestion
}

\author{
TIMOTHY A. SALTHOUSE, THOMAS A. KOLDITZ, JEANNE BUMBERRY, \\ and MARY BETH JOHNSTON \\ University of Missouri-Columbia, Columbia, Missouri 65201
}

\begin{abstract}
Three experiments were conducted to find out why subjects ingest substantially more liquid than required when asked to match a visually displayed volume. All three experiments replicated the basic illusion phenomenon, and Experiments 1 and 2 indicated that it was attributable to the subjects' underestimating the volume of liquid in their mouths. Experiment 3 revealed that the illusion was not present with solid substances, but it was evident with liquids when a quite different measurement procedure was employed.
\end{abstract}

A pilot project in our laboratory recently revealed that subjects, when asked to ingest an amount of liquid comparable to that displayed in a transparent container, consistently ingested more than the displayed amount. The discovery that subjects are not very accurate at estimating the volume of liquid in their mouths would not be particularly interesting if the errors made by subjects were unsystematic. However, this is not the case; subjects nearly always underestimate the amount of liquid in the mouth. The phenomenon is therefore a true illusion and not merely a trivial instance of inaccurate judgment.

To our knowledge, this ingestion illusion has not previously been described, and yet it seems to be quite large and reliable. We therefore designed and conducted several experiments to explore the generality of this phenomenon and to identify some of its constraining conditions.

\section{EXPERIMENT 1}

The first experiment attempted to localize the illusion in either an input (estimation) stage or an output (production) stage. A very simple model of the ingestion task can be postulated in which it is assumed that there are two primary stages or processes involved. The first stage consists of the subject attempting to judge the magnitude of the stimulus according to some internal scale, while the second stage involves the subject translating this internal scale value to a response production system and attempting to generate a response of the desired magnitude. This model suggests that the ingestion illusion might be caused by either (1) a defective stimulusestimation stage in which subjects are overestimating the volumes they are supposed to match, or (2) a defective response-production stage in which subjects are underestimating the volume they are generating.

Three conditions were investigated in the experiment, each involving a different combination of a stimulus mode and a response mode. In Condition A, the volumes to be matched were presented in bottles visible to the subject, and the volume estimates were made by the subject ingesting (through a straw inserted into an opaque container) a volume equal to the volume displayed. This condition is identical to the single condition employed in the pilot experiment.

In Condition B, the stimulus mode was the same as in Condition $\mathbf{A}$, but the response mode was different. The volumes to be matched were again presented in bottles visible to the subject, but now the volumes were estimated by the subject's reporting a number whose magnitude was proportional to the volume of each stimulus.

In Condition $\mathrm{C}$, the response mode was the same as in Condition A, but the stimulus mode was different. Subjects again attempted to estimate the volumes by ingesting the appropriate amount of liquid from an opaque container, but the stimuli were present only as numbers indicating the relationship between the stimulus volume and a standard volume.

If the stimulus-estimation stage is the locus of difficulty, the predictions are as follows. First, the initial illusion (to be demonstrated in Condition A) is explained by postulating that the subject consistently overestimates the volume of liquid in the stimulus containers. That is, if a subject estimates that a volume of $20 \mathrm{ml}$ is really $30 \mathrm{ml}$, he will ingest more than the displayed amount, even if the volume ingested corresponds exactly to the volume estimated. Because Condition B involves the same stimulus mode as Condition $A$, the assumption that the stimulus-estimation stage is defective leads to the prediction that the results in Condition $B$ should be similar to those in Condition A. However, since Condition $\mathrm{C}$ involves a different stimulus mode (which presumably minimizes stimulus-estimation problems), we would not expect an illusion error to be evident in the Condition $C$ results.

If the response-production stage is the source of the ingestion illusion, the predictions would be as follows. First, the basic illusion (which should be replicated in Condition $\mathbf{A}$ ) is explained by postulating that subjects are accurate at judging the volumes 
of the stimulus displays but consistently underestimate the volumes ingested into their mouths. That is, if a subject correctly estimates that a stimulus volume is $20 \mathrm{ml}$ and, in attempting to ingest $20 \mathrm{ml}$, he actually ingests $30 \mathrm{ml}$, the overingestion phenomenon could be attributed to an error in response production. Because Condition $C$ involves the same response mode as Condition $\mathrm{A}$, the assumption that the response-production stage is deficient leads to the prediction that the results in Condition $C$ should be similar to those in Condition $\mathrm{A}$, that is, both should exhibit the ingestion illusion. The different response mode in Condition B, which does not require the ingestion of liquids, leads to the expectation that no illusion error should be present in Condition B.

To summarize, both proposed explanations predict an illusion in Condition $\mathrm{A}$. If the stimulus-estimation process is responsible for this illusion, an illusion should also be present in Condition B but not in Condition $C$. On the other hand, if the responseproduction process is responsible, the illusion should be present in Condition C but not in Condition B.

\section{Method}

Subjects. Thirty college students from introductory psychology courses served as subjects in a single session lasting between 20 and $40 \mathrm{~min}$. All subjects were tested individually.

Apparatus. Eight 135-ml (baby-food) jars with 3 to $38 \mathrm{ml}$ of red (to increase the visibility of the volumes) liquid served as the stimulus volumes. Eight opaque containers (1-pint Mason jars covered with plastic tape), each filled with $100 \mathrm{ml}$ of (room temperature) water and covered with a lid containing a straw, served as the ingestion response devices.

Procedure. Each subject estimated eight volumes in each of three conditions. In Condition A, the stimulus volumes were presented to the subject with instructions to ingest an amount equal to that displayed in the stimulus jar. Ingestion amounts were measured by determining the difference between the volume remaining and the initial $100-\mathrm{ml}$ volume in the ingestion jar. In Condition B, the subjects were shown a standard volume $(20 \mathrm{ml})$ which was assigned the value of 20 . The stimulus volumes were then presented with instructions to assign numbers to them such that the ratio of the number to the standard number corresponded to the ratio of the stimulus volume to the standard volume. In Condition C, only the standard volume $(20 \mathrm{ml}$ and assigned the value of 20) was presented to the subject. The stimulus volumes to be estimated were presented in the form of numbers expressing the ratio of the stimulus volume to the standard volume. The subjects' responses, the volumes of water ingested, were measured in the same manner as in Condition A.

The six possible orders of condition presentation were each administered to five subjects. The order of stimulus presentation within each condition for each subject was determined from a random-numbers table.

\section{Results and Discussion}

The mean responses, that is, volumes ingested in Conditions $\mathrm{A}$ and $\mathrm{C}$ and numbers assigned in Condition $B$, for each of the eight stimulus volumes are displayed in Figure 1.

An analysis of variance confirmed the impressions conveyed from the figure; the factors of condition

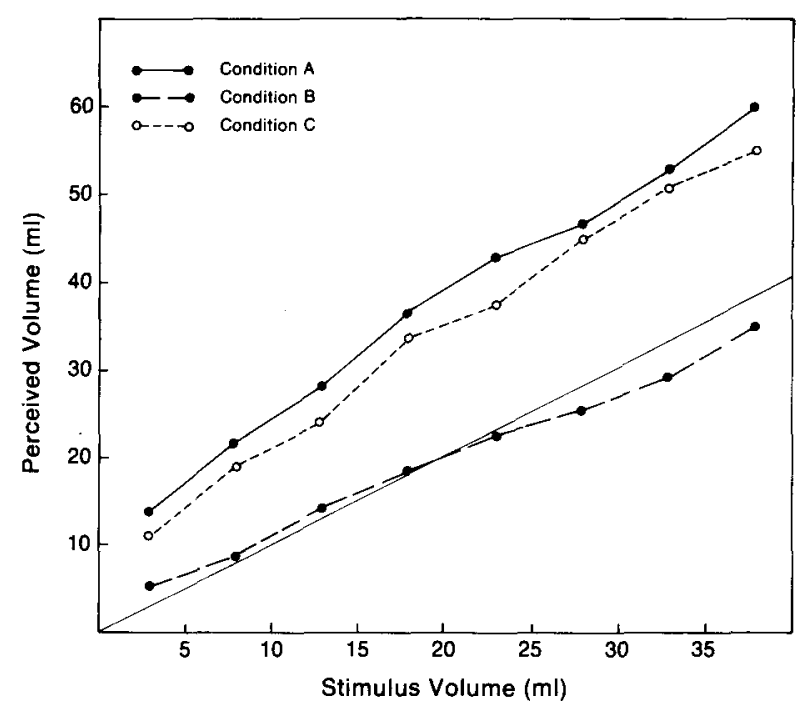

Figure 1. Volume estimates corresponding to the eight stimulus volumes in Experiment 1. Condition $A=$ visual stimulus, ingestion response; Condition $B=$ visual stimulus, numerical response; Condition $\mathbf{C}=$ numerical stimulus, ingestion response.

$[F(2,58)=45.67, p<.0001]$ and volume $[F(7,203)$ $=172.64, \mathrm{p}<.0001]$ were significant, as was their interaction $[F(14,406)=5.62, p<.0001]$. The figure indicates that no illusion was evident in Condition B, but that a substantial overestimation was present in Conditions $\mathrm{A}$ and $\mathrm{C}$. The Condition by Volume interaction reflects the trend for the absolute magnitude of the illusion in Conditions $\mathrm{A}$ and $\mathrm{C}$ to increase with increased volume.

The magnitude of the illusion in Condition $\mathrm{A}$, almost a $200 \%$ overestimation, indicates that the illusion is quite powerful. It is also very reliable, since, of the 30 subjects, the number that ingested more than the displayed volume ranged from $24(80 \%)$ to $29(97 \%)$ across the eight stimulus volumes.

The presence of the illusion in Condition $\mathrm{C}$ but not in Condition B seems to support the hypothesis that a faulty response-production stage is responsible for the illusion. A change in the response mode, that is, switching from ingestion in Condition $\mathbf{A}$ to verbal magnitude estimates in Condition B, eliminated the illusion, but a change in the stimulus mode, that is, switching from direct visual inspection of the stimulus volumes in Condition $\mathrm{A}$ to an indirect generation of stimulus volumes in Condition C, resulted in approximately the same magnitude of illusion. The conclusion, therefore, is that subjects exhibit an illusion because they consistently underestimate the volume of liquid in their mouths.

\section{EXPERIMENT 2}

A comment made by a subject after Experiment 1 
prompted us to devise Experiment 2. This individual had made very small errors in all conditions and, when questioned about her strategy, reported that she had had considerable experience in making visualingestion matches because she measured mouthwash in a small container every morning. Although this experience may not have been the primary factor responsible for her relatively accurate performance, the comment led us to consider the role of short-term experience in modifying the illusion.

The experimental design was a pretest-posttest design with four groups of subjects, each receiving a different type of training experience between the two tests. Group A was a control group that merely waited in the laboratory for $5 \mathrm{~min}$ between the two tests. Group B received visual estimation training, in which the subjects were presented with stimulus volumes and learned to assign the correct numerical magnitudes to them. Group $\mathrm{C}$ received active ingestion training, in which subjects actively ingested (sipped through a straw) fixed volumes of liquid and learned to assign the correct numerical magnitudes to them. Group D received passive ingestion training that was similar to active ingestion training except that the tobe-estimated volumes were poured into the subjects' mouths without any active effort of sipping.

Based on the results of Experiment 1, it was expected that Group B would be similar to Group A in not showing any substantial change in illusion from the pretest to the posttest. The earlier results had suggested that the illusion was not attributable to difficulty in stimulus estimation and, thus, that specific experience at estimating visually displayed volumes should not be beneficial.

If short-term experience was effective in establishing a correspondence between vision and ingestion, we would expect the illusion to be much reduced in the posttest of Condition C. A similar reduction might also be predicted in Condition D if the critical component in the illusion was the mere presence of the liquid in the mouth and not the manner in which it entered the mouth.

\footnotetext{
Method

Subjects. Forty introductory psychology college students were assigned, in the order in which they reported to the laboratory, to alternate experimental groups (10 subjects in each group). The subjects were tested individually in a session that lasted approximately $30 \mathrm{~min}$.

Apparatus. The pretest and posttest apparatus was the same as that described in Experiment 1 . Six additional stimulus jars and six additional response jars were used for the training phase. A small funnel with a straw attached to the narrow end was used by the experimenter to pour water into the subject's mouth in the passive ingestion training phase of Condition $D$.

Procedure. The pretest and posttest were the same in all conditions and essentially like Condition $\mathbf{A}$ of the previous experiment. The subjects were presented with the stimulus volumes and instructed to ingest (through a straw) an amount equal to that dis-
}

played. Different random orders of stimulus presentation were used in the pretest and posttest for each subject.

Condition A subjects received no training experience and merely waited for $5 \mathrm{~min}$ between the two tests.

Subjects in Conditions B, C, and D were yoked in the sense that one subject in each group received the same six stimulus volumes in the same sequences during the training phase as a subject in the other two groups. Different subjects within the same condition, however, received different stimulus volumes selected randomly from 1 to $40 \mathrm{ml}$ with the constraints that no two volumes be within $1 \mathrm{ml}$ of each other and that three volumes be below $20 \mathrm{ml}$ and three above $20 \mathrm{ml}$.

In the training trials, Condition B subjects looked at the display of an amount of liquid and then estimated its magnitude; Condition $\mathrm{C}$ subjects sipped a specified amount of liquid through a straw and then estimated its magnitude; and Condition D subjects had the liquid poured into their mouths and then estimated the magnitudes.

The following instructions were given prior to the training phase: "Now we want you to try to learn to judge the amount of water more accurately. We will present you with different amounts of water and we want you to tell us how much was presented. Six different amounts from $1 \mathrm{ml}$ to $40 \mathrm{ml}$ will be presented, and they will be the same across all training trials. We will tell you the correct answer after you make your response."

The training phase continued for six trials, that is, six presentations of each of the six training volumes, after which the subjects were administered the posttest. The mean accuracy on the last training trial was $70 \%$ for Condition B, $57 \%$ for Condition C, and $55 \%$ for Condition $D$.

\section{Results and Discussion}

The amounts of liquid ingested in the pretest and posttest for the four groups of subjects are displayed in the four panels of Figure 2.

An analysis of variance revealed that the test $[F(1,36)$ $=84.89, \mathrm{p}<.0001]$ and volume $[\mathrm{F}(7,252)=149.66$,

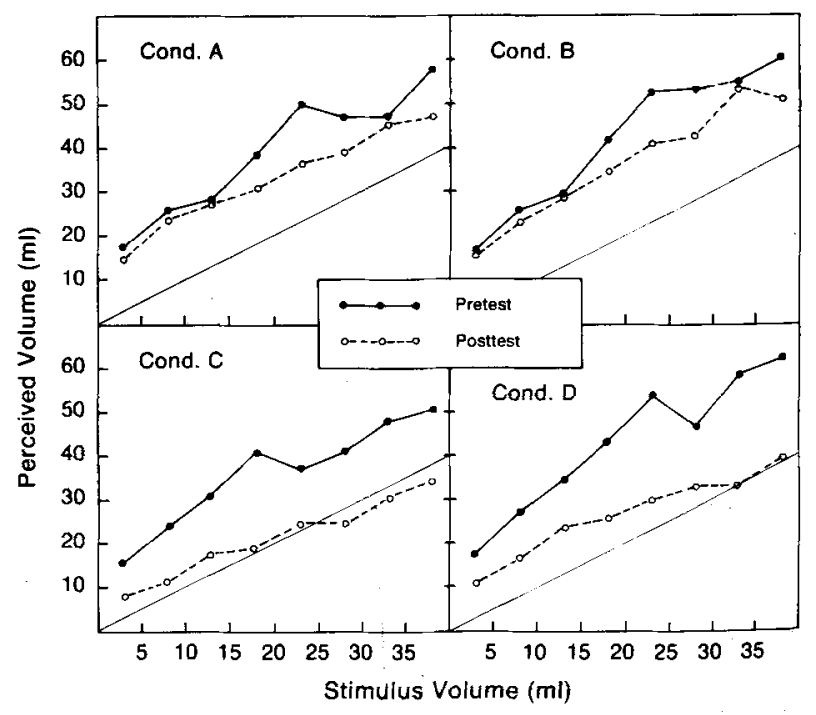

Figure 2. Volume estimates corresponding to the eight stimulus volumes in the pretest and posttest in Experiment 2. Condition $A$ $=$ no training; Condition $\mathrm{B}=$ visual estimation training; Condition $C=$ active ingestion training; Condition $D=$ passive ingestion training. 
$\mathrm{p}<.0001]$ factors and the Condition by Test $[\mathrm{F}(3,36)$ $=5.70, \mathrm{p}<.005]$ and Test by Volume $[F(7,252)$ $=4.77, \mathrm{p}<.0001]$ interactions were all statistically significant. The most interesting of these results is the significant interaction between the condition and test factors. The data illustrated in Figure 2 indicate that this interaction is attributable to a larger change between the pretest and the posttest in Conditions $C$ and D than in Conditions A and B.

As in the previous experiment, the illusion averaged almost $200 \%$ across the pretests in the four conditions. Of the 40 subjects, $33(83 \%)$ to $40(100 \%)$ ingested more than the displayed amount across the eight stimulus volumes.

The large illusion reductions in Conditions $C$ and D but not in Condition B (see Figure 2) serve to confirm and extend the results of Experiment 1. Confirmation of the earlier results is evident in the finding that visual estimation (Condition B training) is not a major factor in the illusion, but that the ingestion process (Conditions $C$ and $D$ ) is an important component. The earlier results are extended in the discovery that the manner in which the liquid enters the mouth (i.e., active sipping in Condition $C$ or passive pouring in Condition D) is apparently an unimportant factor in the illusion.

\section{EXPERIMENT 3}

A final experiment was designed to determine whether the ingestion illusion was an artifact of the particular experimental procedures used or if a different set of procedures would still yield the illusion. In addition, the volumes of both liquids and solids were estimated to find out if the illusion applied to any substance that was in the mouth or if it was limited to liquids.

\footnotetext{
Method

Subjects. Forty introductory psychology students served as subjects in individual sessions lasting approximately $20 \mathrm{~min}$.

Apparatus. Liquids were displayed in $30-\mathrm{ml}$ test tubes and solids in 135-ml transparent jars.

Procedure. The ingestion illusion was measured by placing a liquid or solid in the subject's mouth and then asking him to select (i.e., point to) a match from among a set of 12 displayed volumes. Liquids (water) were poured in the subject's mouth through a funnel. Solids (paraffin balls) were placed in the subject's mouth with a disposable plastic spoon. Neither the liquids nor the solids were visible to the subject. Paper cups were provided for the subjects to dispose of the liquids and solids after making their estimations. The subjects were instructed not to swallow the stimuli but merely to hold them in their mouths until after making the judgment and then to spit the liquid or solid into the paper cup.

The volumes (measured directly with liquids or indirectly by a displacement method with the solids) ranged from 3.3 to $12.7 \mathrm{ml}$ in $1.35-\mathrm{ml}$ steps. The materials displayed for response selection included the actual stimulus volumes plus two smaller volumes $(.3$ and $.8 \mathrm{ml})$ and two larger volumes (14.0 and $15.3 \mathrm{ml}$ ).

Half of the subjects were tested first with the liquids and then
}

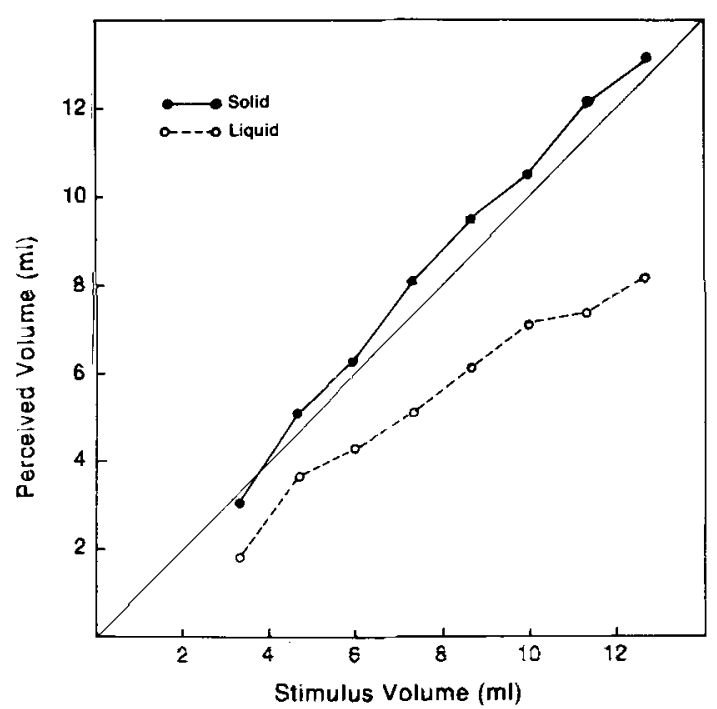

Figure 3. Volume estimates corresponding to the eight stimulus volumes in the solid and liquid conditions in Experiment 3.

the solids; the other half were tested in the reverse order. The order of stimulus presentation for both liquids and solids was determined randomly for each subject.

\section{Results and Discussion}

The mean volumes selected for each stimulus volume are illustrated in Figure 3 for both solids and liquids. In interpreting the figure, it is important to note that if subjects are underestimating the volume in their mouths, as suggested by the results of Experiments 1 and 2, they should select a response volume that is smaller than the stimulus volume. The reversal of the figure compared to Figures 1 and 2 is therefore a consequence of the volumes being placed in the mouth during the stimulus phase rather than serving as the response measure.

The estimations with liquids averaged only about $70 \%$ of the actual volumes, and of the 40 subjects, $23(58 \%)$ to $35(88 \%)$ underestimated the liquids in their mouths. These figures are slightly smaller than those from the previous two experiments, but the illusion is still potent and fairly consistent across subjects.

The estimations with the solids were very accurate and no consistent tendency toward over- or underestimation was apparent.

An analysis of variance revealed that the volume $[F(7,273)=259.62, p<.0001]$ and condition $[F(1,39)$ $=181.54, \mathrm{p}<.0001]$ factors were both significant, as was their interaction $[F(7,273)=17.28, p<.0001]$.

Two conclusions can be drawn from these results. First, the illusion is still evident when using a quite different procedure and, thus, is unlikely to be an artifact of the original experimental procedure. Second, no illusion is exhibited when the substance in the sub- 
ject's mouth is a solid rather than a liquid. This latter fact suggests that the tongue might play an active role in the measurement of substances within the mouth, since only with solid substances could the tongue encounter resistance which would be informative about volume.

\section{GENERAL DISCUSSION}

The experiments described above have demonstrated that there is a large and consistent underestimation of the volume of water in the mouth of naive subjects. Moreover, there is no such underestimation with solid substances, and the liquid underestimation can apparently be corrected with very brief periods of training experience.

Unfortunately, while the preceding statements serve to specify some of the characteristics of the phenomenon, it must be admitted that no explanation can yet be offered to account for the illusion. A general inaccuracy might be expected if subjects had little experience in estimating liquid volumes in the mouth, but the puzzling aspect is that subjects are inaccurate in a consistent direction-they nearly always underestimate the volume in their mouths.

The present phenomenon has a superficial resemblance to Piagetian conservation tasks in that the error produced is nearly always in a specific direction. In conservation tasks, however, the explanation seems to be that children attend to one dimension of the display (e.g., height) to the neglect of another, equally important, dimension (e.g., width). It is not obvious to us how this type of restricted-attention explanation could apply to the ingestion illusion, since there do not appear to be any common dimensions between visual inspection and ingestion of liquid.

It is clear that more speculations, followed by systematic experimentation, are needed to provide a satisfactory elucidation of the illusion of ingestion reported here.

(Received for publication November 27, 1979; accepted February 25, 1980.) 